\title{
CIRCUMSTELLAR MATTER OF YOUNG LOW-MASS STARS: OBSERVATIONS VERSUS THEORY
}

\author{
Claude Bertout \\ Institut d'Astrophysique de Paris \\ 98bis, Boulevard Arago \\ F 75014 Paris \\ France
}

ABSTRACT. After presenting NGC 7129 as a prototypical star-forming region, I discuss what can be learned from the radio spectra of embedded infrared sources. I then review available observational evidence for disks around young stellar objects, with emphasis on accretion disks around $T$ Tauri stars. Finally, new results on the role of magnetic fields in the circumstellar activity of T Tauri stars are presented.

\section{INTRODUCTION}

Observations of molecular clouds offer only an incomplete and unresolved view of what is going on in these star-formation regions, and the large amounts of dust in interstellar clouds alter the spectra of young stellar objects non-linearly. Theoretical ideas about stellar formation must overcome these difficulties.

According to the current pre-main-sequence evolution scenario reviewed of Shu and Adams in this volume, the sequence of events leading to the formation of a low-mass star begins when a rotating molecular core is formed by ambipolar diffusion. This core then undergoes dynamical collapse to make a fast-rotating protostar surrounded by a nebular disk. The central temperature of the protostar increases until it reaches the deuterium fusion ignition point; deuterium burning drives convection within the entire protostar, and thereby turn on dynamo processes which then create a stellar wind. The wind breaks out at the points of least resistance which are the stellar poles where the density of accreting material is lowest. The result is collimated jets and molecular outflows. Finally, the outflow angle widens; the infalling gas is swept out, and the central star and surrounding nebular disk become optically visible. This is the beginning of the T Tauri phase, in which the star is still presumably interacting with its environment. As the strength of this interaction diminishes, the star resembles more and more a normal late-type dwarf.

How observed properties of young stellar objects relate to this theoretical framework is the topic of this review. The first section illustrates 
the current observational status of star-forming regions, using as an example the molecular cloud NGC 7129, which displays most phenomena usually encountered in star formation. In the second section we focus on the radio properties of young stellar objects. Since the Very Large Array allows resolving structures down to about $0 " 1$, radio emission from embedded young objects is a most promising tool for investigating their circumstellar matter. While the observational database at our disposal is much larger for young stars which have become visible in the optical range -- the $T$ Tauri stars -- than for younger objects, various aspects of their activity remain puzzling. Current ideas on the circumstellar environment of $T$ Tauri stars are reviewed in this paper's third and fourth sections, with emphasis on observational clues for the presence of disks and on the role of magnetic activity in the T Tauri phenomenon.

\section{NGC 7129, A STAR-FORMING REGION}

Located in Cepheus at a distance of about $1 \mathrm{Kpc}$ (Racine 1968), the molecular cloud NGC 7129 contains a group of seven early-type stars surrounded by bright reflection nebulosity (Pease 1917). Two of these stars, $\mathrm{BD}+65^{\circ} 1637$ and $\mathrm{LKH} \alpha 234$, were classified as pre-main-sequence stars with approximate spectral types of respectively B5ne and Ae by Herbig (1960).

Every sign of active star formation is present in NGC 7129 . Four Herbig-Haro objects (Strom et al. 1974; Guylbudaghian et al. 1978; Hartigan and Lada 1985) align approximately with LKHo 234 in the NE-SW direction and may be associated with the blue and redshifted $\mathrm{CO}$ molecular gas detected in the region by Edwards and Snell (1983). Several water masers are also found in the vicinity of LKHa 234 (cf. Sandell and 0lofsson 1981) and of a farinfrared source (FIRS; detected by Bechis et al. 1978 ) located 2' South of LKH $\alpha$ 234. Finally, continuum radio emission from NGC 7129 was detected at 23 $\mathrm{GHz}$ by Bertout and Thum (1982) using the Effelsberg $100 \mathrm{~m}$ single dish, and subsequent interferometric observations at the VLA by Snell and Bally (1986) revealed that the radio source was centered on LKHo 234.

With all this activity going on in an area less than $10^{\prime} \times 10^{\prime}$ ', NGC 7129 appears worth a detailed investigation. In particular, the morphology of the Co molecular flows discovered by Edwards and Snell (1983) needs to be clarified. This was the subject of recent observations by Bertout, Cabrit, and Thum (1987) using the IRAM 30m antenna near Granada, Spain. Figures 1 and 2 show preliminary maps of position versus integrated intensity in the ${ }^{12} \mathrm{CO}(\mathrm{J}=1-0)$ line. The $(0,0)$ reference position is that of $L K H \alpha 234$, and offsets are in arc seconds. The beam size is 21", and the mapping has been made at 30" intervals in $\alpha$ and $\delta$.

The first map (Figure 1) shows the ambient $\mathrm{CO}$ gas, as made by integrating the CO line profile over the velocity range corresponding to the static gas. The most striking feature of Figure 1 is the $\mathrm{CO}$ cavity that surrounds the group of hot stars. This hole in the dark cloud is also apparent in optical photographs of the region, where it shows up as an increased density in the number of background stars. The cavity's morphology suggests that it 


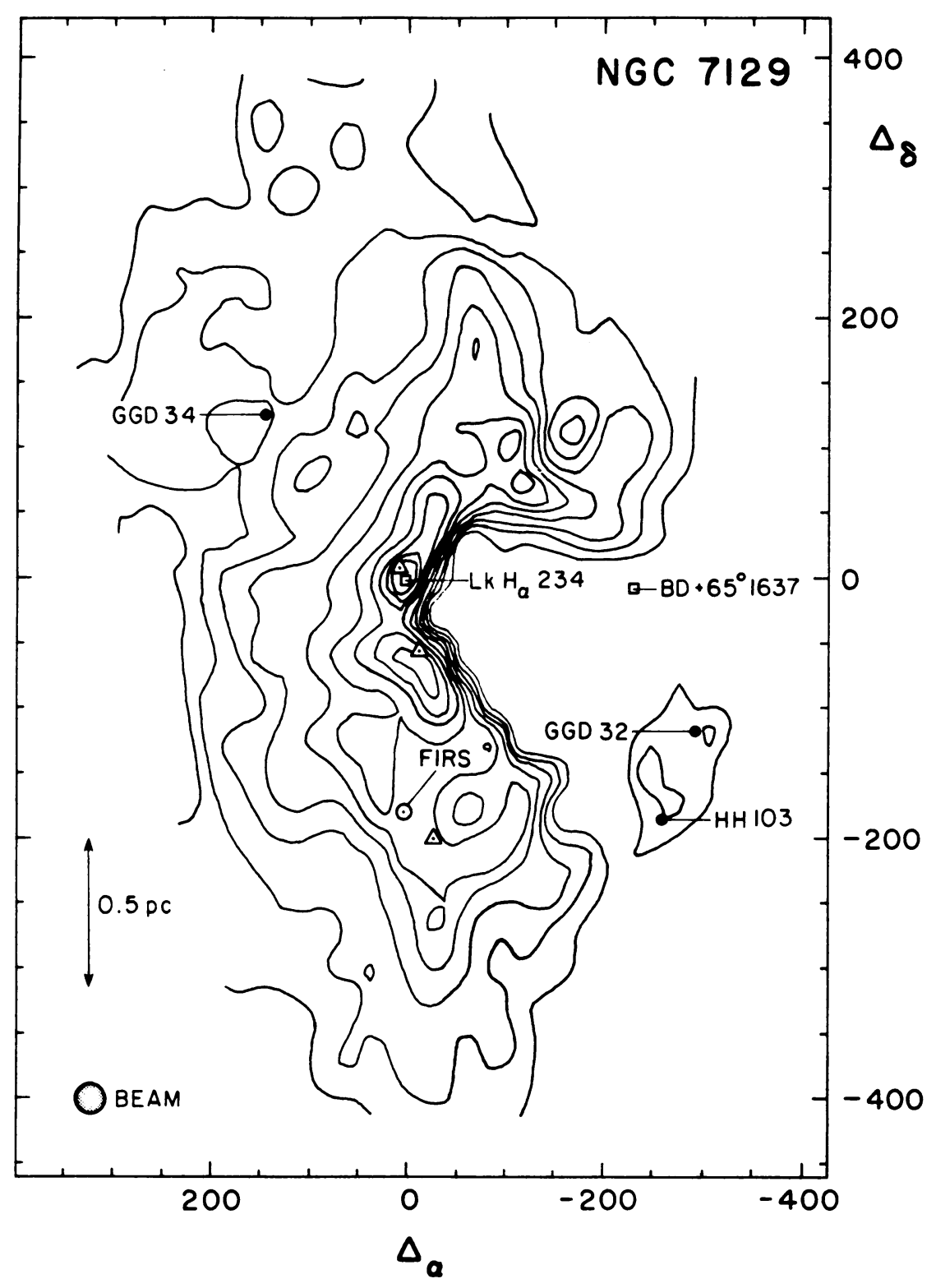

Figure 1 Map of position versus integrated intensity in the $\operatorname{CO}(J=1-0)$ line for the static molecular gas in NGC 7129. Open squares denote two Herbig $\mathrm{Ae} / \mathrm{Be}$ stars, dots mark the positions of Herbig-Haro objects, triangles indicate water masers, and the far-infrared source's position is marked by an open circle. 


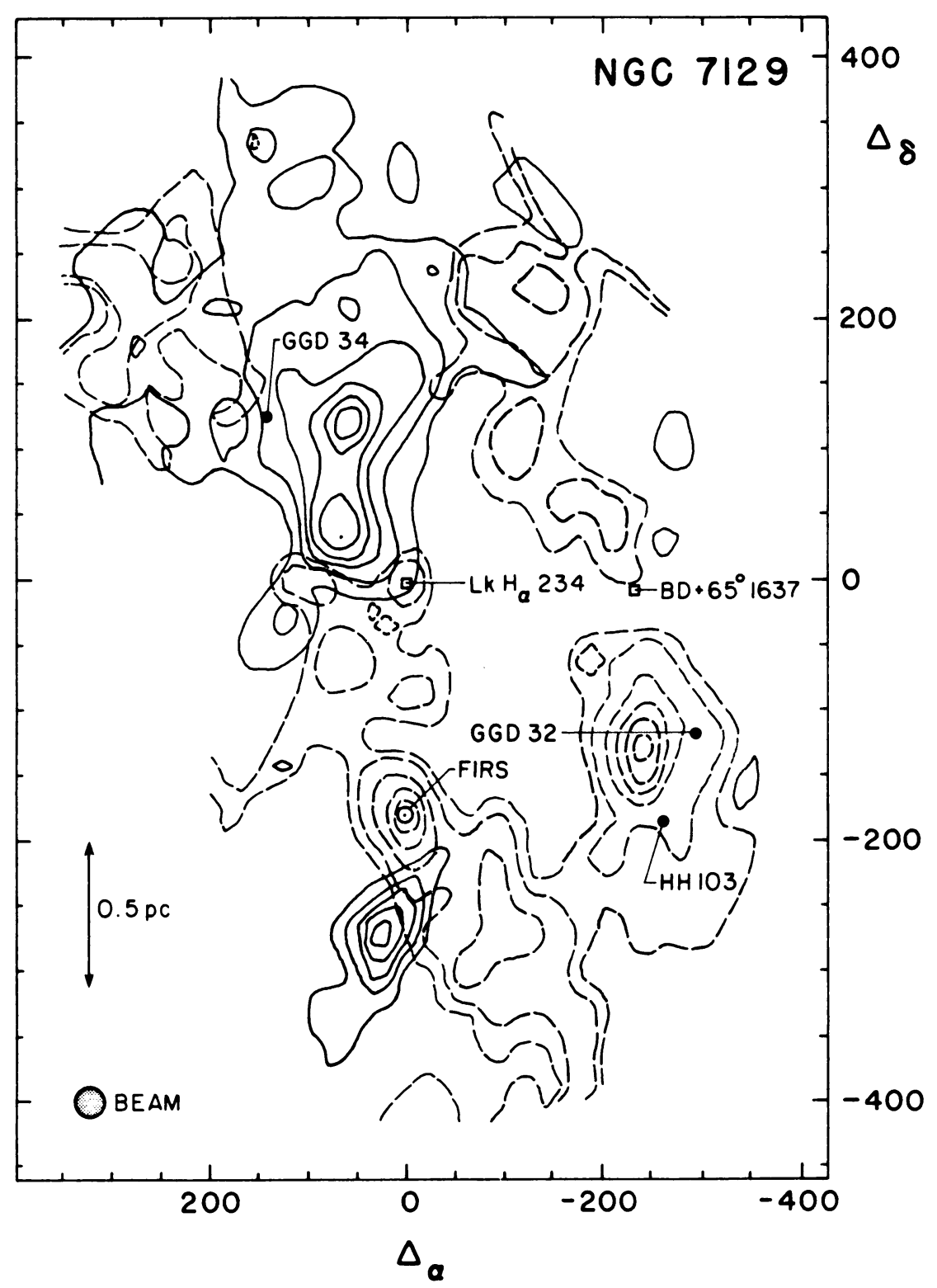

Figure 2 Same as Figure 1, but for the moving CO gas. At least two outflow sources (presumably LKH $\alpha 234$ and FIRS) are present in NGC 7129. 
was created by expansion of a bubble of stellar winds in the direction of least resistance, as suggested by Tenorio-Tagle (1982). Furthermore, both the location of $\mathrm{LKH \alpha}_{\alpha} 234$ at the cavity's apex and the alignment of the cavity's symmetry axis with the Herbig-Haro objects mentioned above suggest that the main stellar wind source is LKHo 234, an assumption consistent with its $H \alpha$ and radio continuum emission.

The complexity of the CO moving gas' map, shown in Figure 2, renders its interpretation difficult. Its main features are an extended, red-displaced emission located NE of LKHa 234 and an extended, weaker blue-displaced emission approximately surrounding the cavity and peaking close to the position of Herbig-Haro objects HH 103 and GGD 32. There is also a nicely symmetric bipolar flow associated with the far-infrared source 2' South of LkHa 234. We show the lowest blue contour at $2 \mathrm{~K} \mathrm{~km} \mathrm{~s}^{-1}$, which is close to the noise level, mainly to demonstrate that the blue-displaced emission closely follows the cavity walls.

A tentative interpretation of the extended red and blue $\mathrm{CO}$ emission calls for a more or less spherically symmetric wind that originates from LkH 234 and that is collimated on a large scale by density gradients in NGC 7129 (cf. Konigl 1982). This is supported both by the map of ambient gas, which reveals that the excitation temperature peaks in a $\mathrm{N}-\mathrm{S}$ elongated region surrounding $L k H \alpha 234$ and by our unpublished CS data. An alternative picture, in which the wind from LKHa 234 is collimated close to the stellar surface by a disk surrounding the star is not supported by the CS interferometric observations of Wilking, Mundy, and Schwartz (1986). The lack of an extended blue lobe is presumably caused by the cavity's containing hot ionized gas flowing out of $L K H \alpha 234$. Full analysis of our $12 \mathrm{CO},{ }^{13} \mathrm{CO}$, and $\mathrm{CS}$ data, now under way, should give us a more precise image of this starforming region.

It has become clear recently that young embedded stellar objects such as LkHo 234 are often radio sources. Snell and Bally (1986) have shown that many infrared sources associated with high-velocity molecular gas are also radio continuum sources. This property gives us access to the inner regions of the flows, which would otherwise be hidden from us by their surrounding dust.

\section{THE RADIO CONTINUUM OF YOUNG STELLAR OBJECTS}

Among the best-investigated radio sources associated with pre-mainsequence objects of low-luminosity ( $\left.L_{\text {bol }}<100 L_{0}\right)$ are IRS5 in L1551, T Tauri South--the infrared and radio companion of the T Tauri optical pair--and the radio source between Herbig-Haro objects 1 and 2 discovered by Pravdo et al. (1985). The radio spectra of these 3 objects are shown in figure 3 . Snell and Bally (1986) studied several additional sources associated with young stellar objects of various luminosities and distinguished two classes of objects. The first exhibits typical stellar wind spectra (spectral index ca. $0.6)$, and the second, which includes L1551 IRS5, has relatively flat spectra which are less easily understood. 


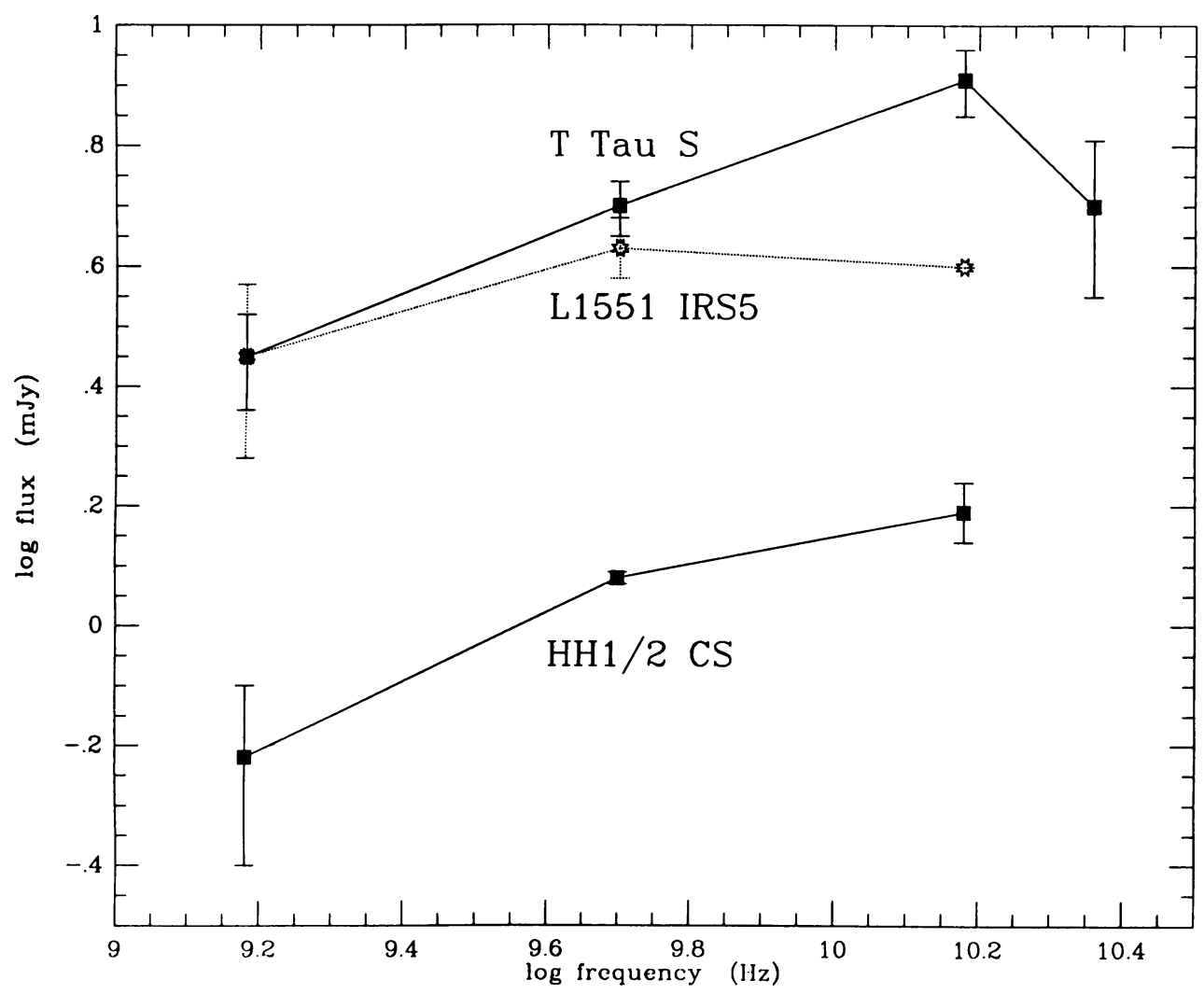

Figure 3 Radio spectra of three embedded low-luminosity sources, as compiled from various sources. The $15 \mathrm{GHz}$ flux of L1551 IRS5 is an upper limit, and the $1.5 \mathrm{GHz}$ data point of T Tauri includes both $\mathrm{N}$ and $\mathrm{S}$ components.

Interpreting the radio spectra of embedded sources assumes that these spectra are thermal. This assumption is justified by the shape of the spectra and by the frequent partial resolution of the radio emitting regions. In the case of T Tauri South, for which more data are available than for other sources, it is also supported by the apparent lack of strong variability on short and intermediate time-scales. This stability contrasts with the radio emission of $T$ Tauri stars, which often show the fast variations and the spectral shapes typical of non-thermal emission (see Montmerle, Andre, and Feigelson 1985). I focus here on radio sources associated with embedded low-luminosity objects, and consider several possible models for spectra that show the qualitative behavior seen in Figure 3: more or less flat spectra at high frequencies with the slope apparently increasing at lower frequencies. 


\section{III.1. Extended Sources}

"Extended" refers here to sources that are partially resolved at $5 \mathrm{GHz}$ by the VLA in A configuration, i.e., to regions with diameter greater than about 0"4, which in turn corresponds to about $60 \mathrm{AU}$ at the distance of the Taurus cloud. After assumption of constant outflow velocity, full ionization and mass conservation in an infinite, spherically symmetric envelope, the emitted radio spectrum varies as $v-0.1$ at frequencies where the envelope is optically thin. The flux is given in a good approximation by (cf. Bertout and Thum 1982)

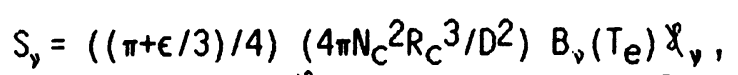

where $D$ is the distance to the star, $X_{y}$ the gas opacity, $R_{C}$ the envelope's inner radius, $N_{C}$ the electron number density at $R_{C}$, and $B_{v}\left(T_{e}\right)$ the $P$ lanck function at the local electron temperature that is assumed constant throughout the envelope. The value of $\epsilon$ is 1 or 2 depending on whether the central core with radius $R_{C}$ is opaque or transparent.

The turnover frequency $v_{t}$ at which the envelope becomes optically thick is given by

$$
\tau_{\nu_{t}}=2 \int_{R_{c}}^{\infty} n_{e}^{2}(r) x_{\nu_{t}} d r \approx 1 \text {, }
$$

Equation 2 then gives the condition on $\mathcal{X}_{\nu}$ at $\nu_{t}$ of

$$
\mathcal{L}_{\nu_{i}} \approx 1 /\left(2 \mathrm{~N}_{\mathrm{C}}{ }^{2} R_{\mathrm{c}}\right) \text {. }
$$

Combining Eqs. 1 and 3 leads to the following relationship between the turnover frequency $\nu_{t}$ and the observed flux $S_{\nu}$ at the same frequency:

$$
S_{\nu_{t}}=(\pi / 2)(\pi+\epsilon / 3)\left(2 k / c^{2}\right)\left(R_{c} / D\right)^{2} \nu_{t}^{2} T_{e} \text {. }
$$

Numbers for a hypothetical "average observed source" can now be inserted into the previous equations. A typical value of the transition frequency $\nu_{t}$ is about $5 \mathrm{GHz}$, and the flux at that frequency is about $10 \mathrm{mJy}$. Assuming $T_{e}=104 \mathrm{~K}$ for the purpose of illustration, Eq. 4 is rewritten as a condition on $R_{C}$ to get $R_{C}=2.210^{14} \mathrm{~cm} \approx 15$ AU. Eq. 2 then gives $N_{C} \approx 810^{5} \mathrm{~cm}^{-3}$, which corresponds to a mass-loss rate of about $410^{-7} \mathrm{M}_{\Theta} / \mathrm{yr}$ when assuming a flow velocity of $300 \mathrm{~km} / \mathrm{s}$. If the spectrum of a partially resolved source at $5 \mathrm{GHz}$ such as IRS5 in L1551 is formed in a spherical, constant velocity wind, it must therefore originate from a region located at a radius of several AU from the star, because of the requirement of optical thinness at frequencies above about $5 \mathrm{GHz}$. This condition means that density cannot increase as $r^{-2}$ down to the surface of the infrared source.

Two possibilities then suggest themselves: (i) the gas flowing away from IRS 5 does not become ionized until it is far from the star, or (ii) our basic assumption of spherical symmetry is inadequate. Little is known about the ionization of outflows from young stellar objects. The number of Lyman photons required to photoionize the gas is, in most cases, far higher than provided by a main-sequence star of bolometric luminosity similar to the 
embedded source (Snell and Bally 1986). But lack of knowledge about the Lyman continuum of pre-main-sequence objects does not allow photoionization to be ruled out. While other mechanisms have been proposed--most notably collisional ionization in the wind (cf. Simon et al. 1983)--, all predict a high degree of ionization close to the central object. The inadequacy of assuming spherical symmetry is therefore a likely possibility, since outflows are often bipolar on intermediate to large scales. In the case of L1551, there is evidence for anisotropy on scales as small as 70 AU (Mundt, 1985). The possibility of a bipolar wind on even smaller scales should thus also be considered.

The present lack of precise estimates for the flow's degree of collimation makes it difficult to predict its detailed emission spectrum when the assumption of spherical symmetry is relaxed (cf. Reynolds 1986). One can check, however, whether the conditions of both small optical depth (necessary to reproduce the shape of the spectrum at high frequencies) and observed flux level can be met for any reasonable choice of main parameters. To do this, it suffices to assume that the flow occurs within two cylinders of radius $R_{d}$ extending from $R_{C}$ to $R_{\max }$ in opposite directions and that the density is constant within the cylinder. The flux in the observer's direction, defined by the view angle $i$ measured from the symmetry axis, is approximately (exactly when $i=90^{\circ}$ ) given by

$$
S_{\nu} \approx(2 \pi / \operatorname{sini})\left(R_{d} / D\right)^{2} N_{0}^{2} \alpha_{\nu} B_{\nu}\left(T_{e}\right)\left(R_{\max }-R_{C}\right)
$$

and as before the condition on optical depth is written as

$$
x_{v_{t}} \approx \operatorname{sini} /\left(2 N_{0} 2 R_{d}\right)
$$

Combining Eqs. 5 and 6 and assuming that $R_{\max } \gg R_{C}$ and that $i=90^{\circ}$, we get a condition on the product $R_{d} R_{\max }$. With the same choice of parameters as before, we find $R_{\max } R_{d} \approx 10^{29} \mathrm{~cm}^{2}$. Since the ionized collimated flow extends at most to about $10^{17} \mathrm{~cm}$ (Mundt 1985), $R_{d}$ is at least of the order $10^{12} \mathrm{~cm}$. Equation 5 then gives $N_{0} \simeq 10^{7} \mathrm{~cm}$, which corresponds to a mass-loss rate of $210^{-10} \mathrm{M}_{\theta} \mathrm{yr}^{-1}$. The derived value of $\mathrm{N}_{0}$ should now be compared to the hydrogen densities in the range $15-130 \mathrm{~cm}^{-3}$ that are typically found in optical jets (Mundt 1986). The discrepancy seems to indicate that the outwards density decrease in the jet is quite strong (optical studies sample primarily the outer parts of the jet), and that the bulk of emission is produced primarily in the innermost, denser ionized regions, where jet collimation is presumably more effective. An outwards decreasing electron density is also necessary in order to account for the low-frequency part of the radio spectrum.

Thus, extended radio emission around young stellar objects probably originates in highly collimated flows (see also Snell et al. 1985). An approximate value of the mass-loss rate derived in the case discussed above is on the order of a few $10^{-10} \mathrm{M}_{\mathrm{O}} \mathrm{yr}^{-1}$, a value apparently much smaller than inferred from the $\mathrm{CO}$ flows associated with these sources. There is, however, considerable uncertainty in mass-loss rates derived from $\mathrm{CO}$ flows (cf. 
Cabrit and Bertout 1986). Alternatively, one can argue either that today's stellar mass-loss is lower than the mass-loss that was once responsible for the large-scale co flow, or that the ionized component represents only a small fraction of the stellar wind's mass.

\section{III.2. Compact Sources}

For unresolved sources, radio spectra with moderate slopes are formed most easily in regions where the density does not fall off too rapidly, e.g. in gravity-driven flows, whether ballistically decelerated outflows or gravitational infall. The gas velocity is $v(r)=V_{c}\left(R_{c} / r\right)^{1 / 2}$, where the sign of $V_{C}$ indicates the flow's direction. Radio observations do not indicate the flow's direction, but only the electron density distribution, which in both cases is given by $n_{e}(r)=N_{C}\left(R_{C} / r\right)^{3 / 2}$ when assuming mass conservation and full ionization. In this case, it is impossible to distinguish between optically thin and thick regimes any more, since the flux varies as -0.1 in both cases (Panagia and Felli 1975). Because the flux integration diverges with infinite envelope radius, one cannot derive a simple formula for the emitted flux equivalent to Eq. 4 above. Instead, one must perform a numerical integration up to a maximum envelope radius. The change in spectral slope is then attributed to the finite size of the envelope rather than to optical depth effects.

This model allows easy reproduction of radio spectra like those of Figure 3 (Bertout 1983). Accretion is thus a viable alternative to winds for explaining the unresolved radio emission of young stellar objects. It is also a physically pleasing one since (i) the gas's potential energy provides a reservoir from which energy needed for ionizing the infalling gas can easily be extracted, and (ii) it is consistent with the scenario of Shu and Adams. Because deviations from spherical symmetry in the accreting material occur close to the star in their model, they would go unnoticed in the radio centimetric spectrum, which samples a region located at $10^{14}-10^{15} \mathrm{~cm}$ from the stellar center.

There is however next to no other observational evidence for gas accretion in young embedded stars such as T Tauri South or L1551 IRS5, which are both associated with molecular $\mathrm{CO}$ outflows. Instead, a spectrogram of IRS 5 obtained by Mundt et a1. (1985) reveals a Ho Type-I P Cygni profile, which further confirms that the ionized gas around the star is flowing out. But then, IRS5 is an extended radio source; and it is conceivable that it represents a later stage than T Tau $S$ in Shu's scenario, in which the emission measure of the outflowing gas would largely dominate emission of accreting material. At any rate, results are the same for ballistic outflow and accretion flow. With the same input data as above, one finds the following representative values for the parameters of the radio emitting regions: $R_{C} \approx$ $2 R_{\theta}, R_{\max } \approx 510^{3} R_{C}, N_{C} \approx 310^{10} \mathrm{~cm}^{-3}$, which correspond to a mass-flow rate of approximately $610^{-9} M_{\odot} y r^{-1}$ when assuming $V_{C}=300 \mathrm{~km} \mathrm{~s}^{-1}$. 
Both spherical gravity-driven flows and well-collimated, bipolar winds will thus emit radio spectra similar to those observed. Resolution of the source (or lack thereof) provides one means of distinguishing between these possibilities, as do high signal-to-noise measurements of the radio flux at centimetric wavelengths. The two models, which produce similar (but distinguishable, at least in principle) spectra, may correspond to different phases in the scenario described by Shu and Adams, with the unresolved sources still in their accretion-dominated phase while resolved sources have already developed a sizable wind.

\section{EVIDENCE OF DISKS AROUND YOUNG STARS}

\section{IV.1. Large-Scale Structures}

Young stellar objects are embedded in molecular clouds, and their light is absorbed and reemitted in the far-infrared by the cloud's dust. The performance of available infrared detectors makes the direct detection of disks around these objects a rather difficult task. The work of Cohen et al. (1985) demonstrates both the successes and still present limitations of far-infrared observations using airborne observatories. Observations of molecular lines at radio wavelengths also reveal somewhat flattened structures around young, massive stellar objects. But the relationship, if any, between these large-scale structures and the underlying nebular disks discussed by Adams and Shu is totally unclear at this point.

Observations offer indirect evidence for the presence of a disk around the T Tauri star HL Tauri. Grasdalen et al. (1984) recorded digital images of $\mathrm{HL}$ Tauri at 1.6 and $2.2 \mu$ and used maximum entropy image reconstruction techniques to show that the star was surrounded by a somewhat flattened structure that they interpret as light scattered by dust in a disk with diameter about 300 AU surrounding HL Tau. Beckwith et al. (1986) used the Owens Valley millimetric interferometer to map the $\mathrm{CO}(\mathrm{J}=1 \rightarrow 0)$ transition in the vicinity of $\mathrm{HL}$ Tau, and conclude that the star is embedded in a smallscale (<900 AU) concentration of molecular gas which appears gravitationally bound to the star. Beckwith et al. suggest that the condensation represents a disk with some $0.01 \mathrm{M}_{\odot}$.

\section{IV.2. Small-Scale Accretion Disks Around T Tauri Stars}

The infrared excess observed in T Tauri stars and related objects has traditionally been attributed to thermal reemission of stellar photons by $a$ dusty envelope (Mendoza 1968; Cohen and Kuhi 1979). And ultraviolet excess emission is usually thought to be of chromospheric origin (cf. Herbig and Goodrich 1986). The physical processes responsible for chromospheric and envelope heating have not yet been determined, but magnetism has been suggested by several authors. Instead, thermal emission from a dusty accretion disk surrounding the young star provides a more attractive and selfconsistent explanation of both the near-infrared and ultraviolet excesses observed in T Tauri stars. 
The theoretical structure and evolution of steady-state accretion disks was investigated by Lynden-Bell and Pringle (1974), who find that the temperature distribution $T_{D}(r)$ of an accretion disk surrounding a central star of mass $M_{*}$ and radius $R_{*}$ is related to accretion rate $M$ by

$$
T_{D}(r)=\left(\left(3 G M_{*} \dot{M} / 8 \pi \sigma r^{3}\right)\left(1-\left(R_{*} / r\right)^{1 / 2}\right)\right)^{1 / 4}
$$

where $G$ is the gravitational constant and $\sigma$ the Stefan-Boltzmann constant. Provided the star rotates slower than the inner part of the disk, about half of the accretion energy $E_{k}=G M_{*} \dot{M} / 2 R_{*}$ is radiated away in a boundary layer near the central star, with temperature

$$
T_{B} \approx 6.510^{4}\left(M_{*} \dot{M} / 10^{-5} M_{\Theta} y r^{-1}\right)^{1 / 4}\left(R_{*} / R_{\Theta}\right)^{-3 / 4}
$$

The emergent spectrum can then be calculated from these temperature laws. Several investigators have done this for accretion disks in cataclysmic variables and symbiotic stars and, more recently for FU Orionis objects (Hartmann and Kenyon 1985). Because of the large range of system temperatures, the spectrum exhibits both ultraviolet and infrared excesses over the energy distribution of the central star alone. I am investigating the disk hypothesis for $T$ Tauri stars using a database of photometric measurements gathered simultaneously in the optical (UBVRI filters) and in the nearinfrared (JHKL filters). Examples of computed spectra are given in Figure 4 along with the observed energy distributions of four T Tauri stars. Besides the view angle, the main free parameters are (i) the disk's maximum temperature $\mathrm{Td}_{\max }$ ( $\mathrm{ii}$ ) the stellar radius, and (iii) the outer disk radius. These preliminary results are summarized in Table 1, where both the stellar effective temperature and the visual extinction are consistent with Cohen and Kuhi's (1979) data, while the four other disk parameters have been adjusted to make a reasonably good fit to the data. The lack of IUE and far-infrared data does not allow us to constrain the model entirely, however. In particular, far-infrared data are needed for testing the disk's temperature distribution more exactly, which is an important issue since one expects differeit temperature distributions for different disk support mechanisms (cf. Adams, Lada and Shu 1987). The nebular disks advocated by Adams and Shu do not emit strongly in the ultraviolet, since they have no accretion boundary layer (the stellar photosphere is assumed to rotate as rapidly as the inner parts of the disk). While this may be true for embedded sources, we know that the photospheres of $T$ Tauri stars rotate slowly (cf. Bouvier et al. 1986). It is therefore conceivable that an accretion boundary layer is formed only after the central star has lost angular momentum, presumably as a result of its strong wind.

There have been few attempts to find evidence of disks in line spectra of young stellar objects, mainly because it requires high-resolution spectroscopy of rather faint objects. Hartmann and Kenyon (1985) note that some absorption lines of $V 1057$ Cygni are double, which they attribute to rotation in the accretion disk. Another indirect indication of the presence of disks around T Tauri stars is also the shape of their forbidden lines formed in the moving gaseous envelopes surrounding the stars. Appenzeller et 21. (1984) compare high-resolution ( $R>10^{4}$ ) profiles of forbidden lines in $T$ Tauri stars with predictions from model computations using several geomet- 

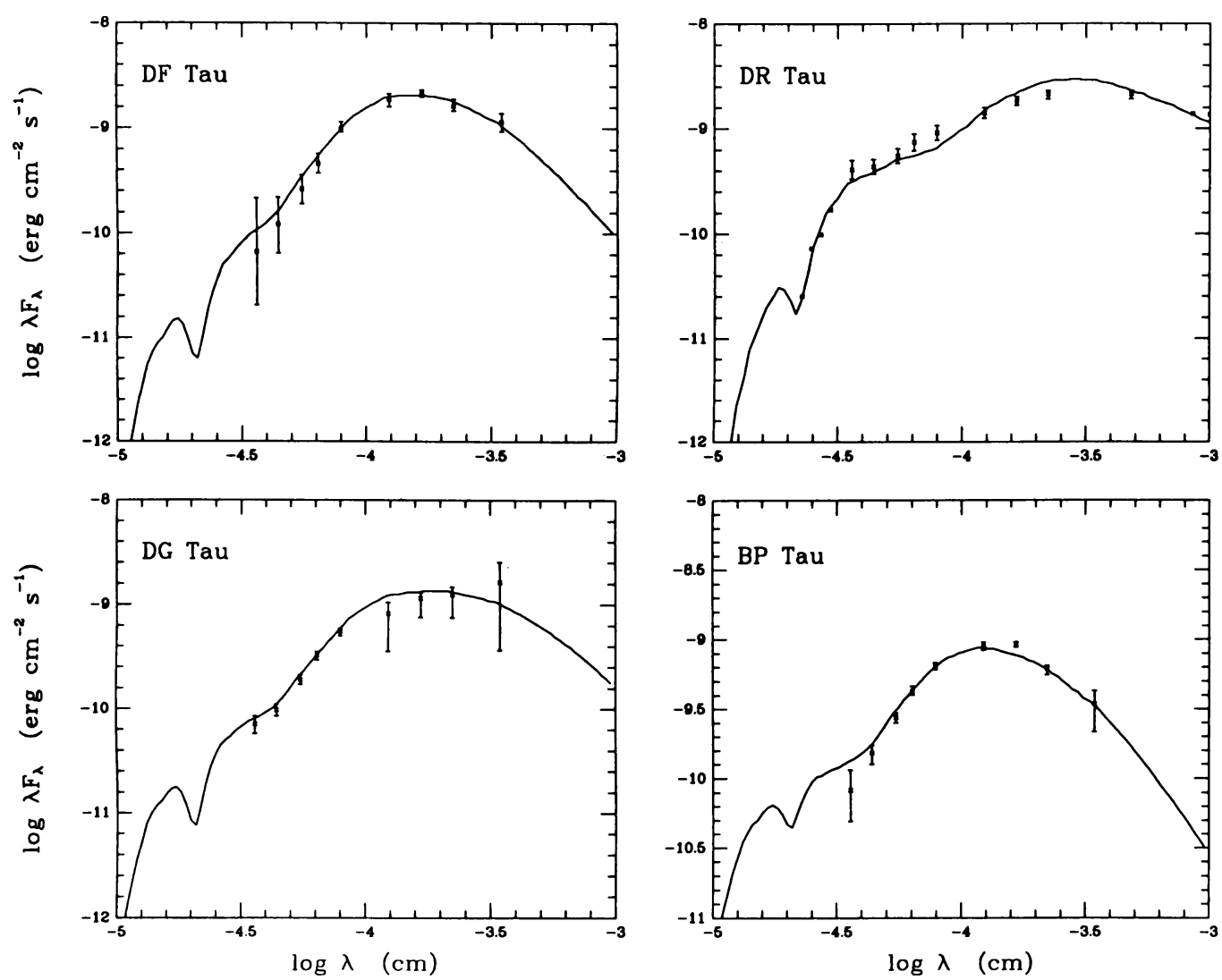

Figure 4 Preliminary comparison of accretion disk spectra with observed energy distributions of T Tauri stars. Error bars on the photometric data do not represent photometric accuracy, but indicate instead the amplitude of observed variations (when available). In the case of DR Tauri, a rather extreme $T$ Tauri star, the bars show typical night-to-night variations.

Table 1 Disk model parameters

\begin{tabular}{lcccccc} 
Star & $\begin{array}{c}R_{*} \\
\left(R_{0}\right)\end{array}$ & $\begin{array}{c}R_{\text {disk }} \\
\left(R_{0}\right)\end{array}$ & $\begin{array}{l}T_{\text {dmax }} \\
\left(R_{\max }\right)\end{array}$ & $\begin{array}{c}i \\
\left({ }^{\circ}\right)\end{array}$ & $\begin{array}{c}\text { Teff } \\
(K)\end{array}$ & $\begin{array}{c}A_{V} \\
(\text { mag })\end{array}$ \\
\hline DF Tau & 3 & 6 & 2700 & 40 & 3800 & 2.0 \\
DR Tau & 4 & 50 & 2400 & 0 & $<2500$ & 1.9 \\
DG Tau & 2 & 50 & 3500 & 75 & 4100 & 1.4 \\
BP Tau & 3 & 5 & 2300 & 75 & 3800 & 0.9
\end{tabular}


ries for the emission region; and they find that the often blue-displaced forbidden lines are formed not in spherically symmetric flows, but rather in cones. Furthermore, the presence of a dusty disk around the star appears necessary to explain occultation of the red side of the line.

\section{MAGNETISM AS THE ENGINE OF T TAURI CIRCUMSTELLAR ACTIVITY}

More than just a disk is needed to explain the multiciplity of phenomena observed in the circumstellar environment of T Tauri stars (cf. Bertout 1984 for a review). While disks provide a geometry which helps collimate and ionize outflows, it is unlikely that they are actually driving the flows. Bouvier (1986) studied correlations between various emission lines that are known diagnostics of magnetic activity in late-type dwarfs. An unexpected result of this investigation is that the surface fluxes (and total luminosities) in the $H \alpha$, CaII $K$ and MgII $k$ lines correlate rather well with one another for a large sample of $T$ Tauri stars of different activity levels. Furthermore, these correlations apparently match the correlations found in main-sequence stars (see the contribution by Bouvier in this volume). These results are not fully understood at this point. It seems unlikely that both $K$ and $H \alpha$ are formed in exactly the same atmospheric region, since (i) their excitation mechanisms are usually different in late-type dwarfs, and (ii) their profile widths and shapes are usually quite different too. They may, however, be formed in different parts of the same overall region, such as in a stellar wind, while the previous correlation indicates that a common underlying physical process is responsible for both lines. It is nevertheless curious that both $\mathrm{H \alpha}$ and CaII flux should scale in exactly the same way when the strength of the underlying process, presumably the magnetic field, varies. A detailed, quantitative interpretation of these intriguing data is likely to bring valuable insight in the structure of $\mathrm{T}$ Tauri envelopes.

Studies of $X$-ray emission as a function of stellar rotation and of spot properties in $T$ Tauri stars support the view that part of $T$ Tauri activity is magnetically driven. Figure 5 demonstrates that the X-ray flux of $T$ Tauri stars is anti-correlated, albeit weakly, with the rotation period (Bouvier 1986). Such a relationship is also found in other late-type stars, and is usually interpreted as an indication for dynamo-driven coronal heating. Basri, Laurent and Walter (1985) show that the X-ray surface flux of RS CVn stars is not as tightly correlated with rotation period as other activity indicators. A correlation study of UV lines and rotation in T Tauri stars would therefore help confirm the correlation in Fig. 5. Periodic light curves variations--interpreted as rotational modulation by groups of spots on the stellar surface--are found in the whole population of $T$ Tauri stars irrespective of their place in the HRD and of their activity level (Bertout, Bouvier and Bouchet 1987). Computed properties of spots show them to be quite similar to spots found on RS CVn systems. This further confirms that rotational velocity, which is quite similar in $T$ Tauri stars and $R S$ CVn stars, is the main parameter in stellar magnetic activity. 


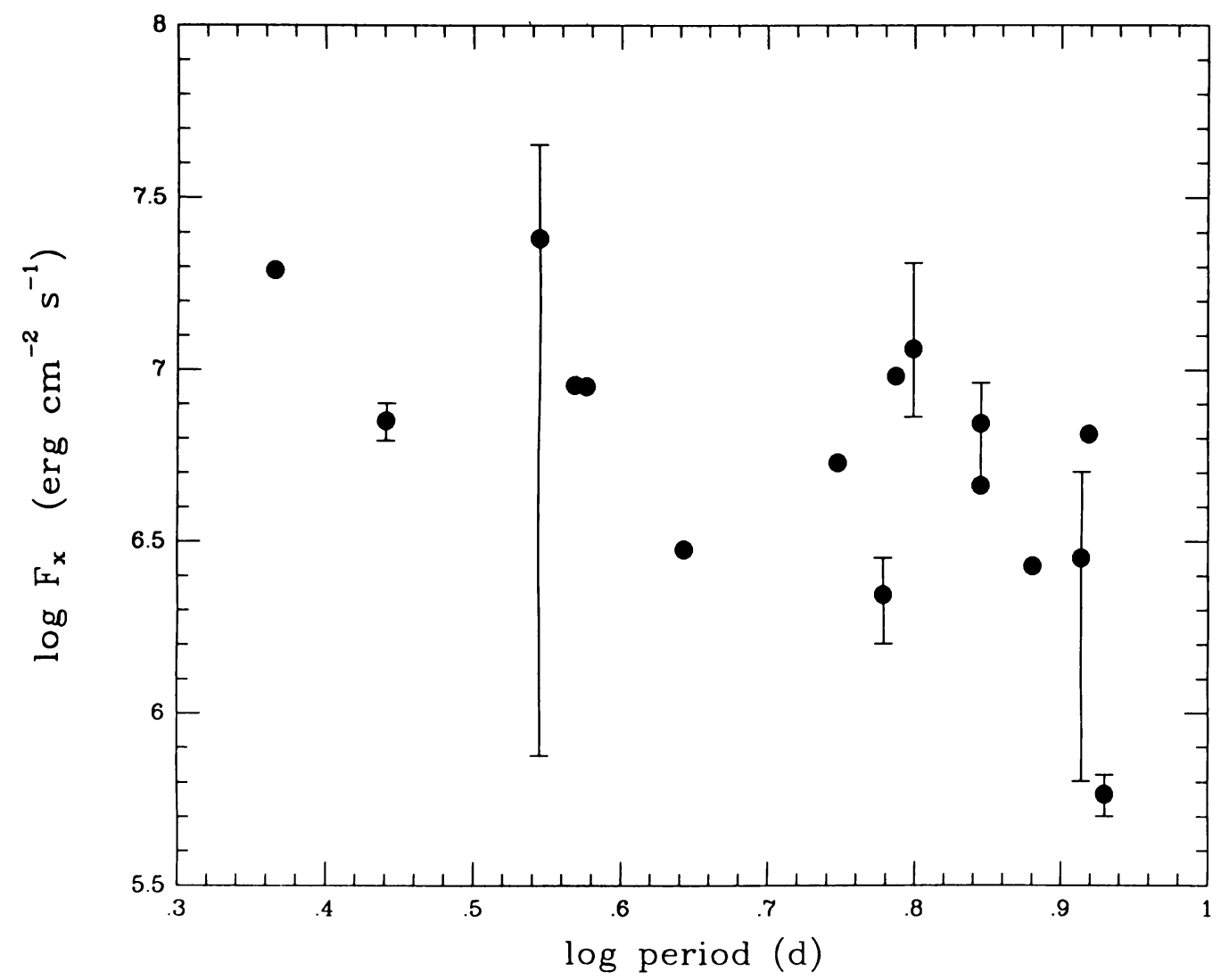

Figure 5 The relationship between $X$-ray flux and rotation period of T Tauri stars. The observed range of flux variability is indicated by bars when several measurements have been made; in this case, the dots indicate the most often observed flux value.

\section{SUMMARY}

The main conclusions of this review are as follows.

i) Compact radio sources associated with young stellar objects may provide evidence for protostellar accretion. High signal-to-noise VLA spectra and direct use of visibility functions would allow testing this possibility further.

ii) More extended radio emission like that from IRS5 L1551 is best interpreted as caused by a bipolar collimated wind, and may represent a somewhat later evolutionary phase than compact sources.

iii) Disks are probably present around many embedded low-luminosity infrared sources and T Tauri stars, and evidence for this is discussed both in Shu and Adams' review and above. While one can argue about the detailed 
nature of these disks, it is encouraging for future quantitative work that the structure of disks has already been studied in several other astrophysical instances.

iv) Presence of a disk and magnetically-driven atmospheric activity appear to be major ingredients of the T Tauri phenomenon. Their respective roles must be further disentangled.

Many features of young objects --radio emission, bipolar flows, magnetic activity-- were not predicted by theories of star formation. This does not necessarily mean that these theories are at fault, since the appearance of young stars is determined mainly by circumstellar and surface phenomena. Indeed, theorists are doing their best to catch up with observations.

CREDITS It is a pleasure to thank Sylvie Cabrit and Jerôme Bouvier for their contributions to Sections II and $V$ respectively. I also enjoyed valuable discussions with Philippe Andre and received considerable help from Joli Adams in editing the text.

\section{REFERENCES}

Adams, F., Lada, C.J., Shu, F.: 1987, Astrophys. J., in press Appenzeller, I., Jankovics, I., Oestreicher, R.: 1984, Astron. Astrophys. 141,108

Basri, G., Laurent, R., Walter, F.M.: 1985, Astrophys. J. 298, 761

Bechis, K.P., Harvey, P.M., Campbel1, M.F., Hoffmann, W.F.: 1978, Astrophys. J. 226,439

Beckwith, S., Sargent, A.I., Scoville, N.Z., Masson, C.R., Zuckerman, B., Phillips, T.G.: 1986, preprint

Bertout, C.: 1983, Astron. Astrophys. 126, L1

Bertout, C.: 1984, Rep. Prog. Phys. 47, 111

Bertout, C., Bouvier, J., Bouchet, P.: 1987, in preparation

Bertout, C., Cabrit, S., Thum, C.: 1987, in preparation

Bertout, C., Thum, C.: 1982, Astron. Astrophys. 107, 368

Bouvier, J. 1986: Doct. Thesis, Paris VII University, Paris, France

Bouvier, J., Bertout, C., Benz, W., Mayor, M.: 1986, Astron. Astrophys., 165,110

Cabrit, S., Bertout, C.: 1986, Astrophys. J., 307, 313

Cohen, M., Kuhi, L.V.: 1979, Astrophys. J. Suppl. 41, 743

Cohen, M., Harvey, P.M., Schwartz, R.D.: 1985, Astrophys. J. 296, 633

Edwards, S., Snell,R.L.: 1983, Astrophys. J. 270, 605

Grasdalen, G.L., Strom, S.E., Strom, K.M., Capps, R.W., Thompson, D., Castelaz, M.: 1984, Astrophys. J. Letters 283, L57

Guylbudaghian, A.L., Glushkov, Y.J., Denisuyk, E.K.: 1978, Astrophys. J. Letters 224, L127

Hartigan, P., Lada, C.J.: 1985, Astrophys. J. Suppl. 59, 383

Hartmann, L., Kenyon, S.J.: 1985, Astrophys. J. 299, 462

Herbig, G.H.: 1960, Astrophys. J. Suppl. 4, 337

Herbig, G.H., Goodrich, R.W.: 1986, Astrophys. J., in press

Konig1, A.: 1982, Astrophys. J. 261, 115 
Lynden-Bell, D., Pringle, J.E.: 1974, Mon. Not. R. astr. Soc. 168, 603 Mendoza, V.E.E.: 1968, Astrophys. J. 151, 977

Montmerle, T., Andre, P., Feigelson, E.: 1985 in Nearby Molecular Clouds,

Ed. G. Serra, Lectures Notes in Physics 237, Springer-Verlag Heidelberg

Mundt, R.: 1985 in Nearby Molecular Clouds, Ed. G. Serra, Lectures Notes in Physics 237, Springer-Verlag Heidelberg

Mundt, R.: 1986, Canadian Journal of Physics, in press

Mundt, R., Stocke, J., Strom, S.E., Strom, K.M., Anderson, E.R.: 1985, Astrophys. J. Letters 297, L41

Panagia, N., Felli, M.: 1975, Astron. Astrophys. 39, 1

Pease, F.G.: 1917, Astrophys. J. 46, 24

Pravdo, S.H., Rodriguez, L.F., Curiel, S., Canto, J., Torrelles, J.M., Becker, R.H., Sellgren K.: 1985, Astrophys. J. Letters 293, L35

Racine, R.: 1968, Astron. J. 73, 233

Reynolds, S.P.: 1986, Astrophys J. 304, 713

Sandel1, G., Olofsson, H.: 1981, Astron. Astrophys. 99, 80

Simon, M., Felli, M., Cassar, L., Fischer, J., Massi, M.: 1983, Astrophys. J. 266,623

Snell, R.L., Bally, J.: 1986, Astrophys. J., 303, 683

Snell, R.L., Bally, J., Strom, S.E., Strom, K.M.: 1985, Astrophys. J. 290, 587

Strom, S.E., Grasdalen, G.L., Strom, K.M.: 1974, Astrophys. J. 191, 111

Tenorio-Tagle, G.: 1982, in Reqions of Recent Star Formation, Eds. R.S. Roger and P.E. Dewdney (Dordrecht: Reidel), p. 1

Wilking, B.A., Mundy, L.G., Schwartz, R.D.: 1986, Astrophys. J. Letters 303, L61 